\title{
Relationship Between Maternal Clinical Factors and Mother-Reported Child Problems
}

\author{
Carol T. Mowbray, Ph.D. \\ Lisa Lewandowski, Ph.D. \\ Deborah Bybee, Ph.D. \\ Daphna Oyserman, Ph.D.
}

\begin{abstract}
Maternal depression has been associated with mothers' elevated reports of child problems. However, it is unclear the extent to which elevations in mother ratings reflect having a depression diagnosis, having any mental illness diagnosis, or having a diagnosis vs. symptom levels. As part of a NIMH-funded, longitudinal study of mothers with serious mental illness $(N=379)$, we examined the relationship between mother-reported adolescent behavior problems $(N=78)$ and maternal depression vs. other diagnoses, as well as the effects of depression diagnosis vs. symptom levels. Mothers were recruited from the public mental health system in an urban area, and are primarily African-American and low income. We found that maternal psychiatric symptoms made a unique and significant contribution to explaining the variance in mother-reported child problems, independent of controls (e.g., teacher reports and child demographics), while maternal diagnosis did not. Implications of findings are discussed.
\end{abstract}

KEY WORDS: mothers; mental illness; child problems; depression; psychiatric diagnosis.

This study was funded by NIMH grants awarded to Carol T. Mowbray (\#R01MH54321) and Daphna Oyserman (\#R01MH57495).

Carol T. Mowbray and Lisa Lewandowski are affiliated with School of Social Work, University of Michigan, 1080 South University, Ann Arbor, MI 48109-1106.

Deborah Bybee is affiliated with Department of Psychology, Michigan State University, East Lansing, MI.

Daphna Oyserman is affiliated with School of Social Work, Department of Psychology, and Institute for Social Research, University of Michigan, Ann Arbor, MI.

Address correspondence to Daphna Oyserman, Institute for Social Research, University of Michigan, Ann Arbor, MI; e-mail: daphna@umich.edu. 


\section{INTRODUCTION}

\section{Relationship Between Maternal Clinical Factors \& Mother-Reported Child Problems}

The relationship between maternal depression and mother-reported problems of children and adolescents has been the subject of extensive research. While some studies suggest that maternal depression does not prevent mothers from providing accurate reports of child behaviors (Ackermann \& DeRubeis, 1991; Conrad \& Hammen, 1989; Haaga \& Beck, 1995; Querido, Eyberg, \& Boggs, 2001), others suggest that depression-related distortions exist (Fergusson, Lynskey, \& Horwood, 1993; Najman et al., 2001; Youngstrom, Loeber, \& Stouthamer-Loeber, 2000; Youngstrom, Izard, \& Ackerman, 1999). Recent research seems clear, however, in providing evidence that mothers with depression do report elevated levels of child problems. That is, in comparison studies of maternal vs. child or teacher reports, ratings of depressed mothers have been shown to be significantly elevated concerning child externalizing and internalizing behaviors, total problem behaviors, and symptoms of conduct disorder or hyperactivity (Boyle \& Pickles, 1997a; Chilcoat \& Breslau, 1997; Najman et al., 2000; Youngstrom et al., 2000). Additionally, Youngstrom et al. (1999) found dysphoria-related elevations in maternal reports of negative child behaviors relative to those of independent observers.

The purpose of this article is to present research on the relationship between maternal ratings of the behavior of adolescent children and maternal mental illness for adolescents whose mothers have diagnosed psychiatric disorders-depression as well as bipolar and schizophreniaspectrum disorders. The results to be reported should make a contribution to the literature by examining the extent to which elevations in mother ratings relate to depression vs. other diagnoses, and the effects of diagnosis vs. symptom levels, while controlling for child demographics and teacher ratings.

\section{Measures of Maternal Depression}

In most research reported, maternal depression has been based on symptom measures from "normal" populations, and not from women with depression diagnoses. That is, symptom checklists have been used as opposed to standardized diagnostic instruments. For example, Youngstrom et al. (2000) created a symptom/severity scale based on 
DSM criteria; Youngstrom et al. (1999) measured dysphoria using the BDI Differential Emotions Scale, State-Trait Anxiety Inventory, and observer ratings of maternal emotional expression; and Boyle and Pickles (1997b) used the CES-D and Negative Affect Balance Scale to assess mothers' depression symptoms. With the exception of several early studies (Conrad \& Hammen, 1989; Richters \& Pellegrini, 1989) and, more recently Chilcoat and Breslau (1997), research has not included clinical samples of mothers nor used major depression diagnosis as the measure of maternal depression. Haaga and Beck (1995) argued for greater inclusion of participants with a diagnosis of depression in order to insure that more severe cases are not excluded and to avoid the overlap commonly found between symptom measures of anxiety and depression. In fact, studies using multiple measures of depression suggest that depressive symptoms rather than depression history or lifetime diagnosis are related to elevations in maternal reports of child externalizing, internalizing, or total problems (Hammen et al., 1987; Najman et al., 2000). This is in contrast to early studies (Conrad \& Hammen, 1989; Richters \& Pellegrini, 1989) that found no depressionrelated elevations in maternal reports regardless of the depression measure used. The absence of significant results in these studies, however, may be due to small sample sizes.

\section{Other Maternal Clinical Indicators}

Some research has investigated the effects of other psychiatric symptoms in addition to, or instead of depression, in relationship to motherelevated reports of child problems. Thus, mother-reported elevations in child internalizing (relative to child reports) have been associated with total maternal psychiatric symptom levels (as measured by the total SCL-90; Kolko \& Kazdin, 1993) and with combined depression and anxiety scores (subscales of the Delusions-Symptoms States Inventory; Najman et al., 2001). Symptom-related elevations in maternal reports relative to both child and spouse reports were also found using the Brief Symptom Inventory (BSI) (Treutler \& Epkins, 2003). Additionally, compared to child reports, maternal-reported elevations in child externalizing were associated with symptoms of both anxiety and depression (Najman et al., 2001). Frick, Silverthorn, and Evans (1994) found that maternal anxiety symptoms and anxiety disorder diagnosis related to elevations in maternal-reported child anxiety. Thus, there is evidence of elevations in maternal reported child problems being associated with psychiatric symptoms other than depression. 
However, few studies have compared the impact of maternal depression vs. other maternal psychiatric symptoms on mother-reported child problems. Briggs-Gowan, Carter, and Schwab-Stone (1996) looked at both the unique and shared contributions of maternal anxiety (Health Opinion Survey) and depression (CES-D) to the variance in mother-reported child problems. They found that general distress rather than depression alone was related to elevations in maternal reports of daughters' externalizing symptoms relative to teacher or child reports. On the other hand, a longitudinal analysis conducted by Renouf and Kovacs (1994) found depression-related elevations in motherreports of child depression over time (relative to child report), but no effect of overall maternal symptom levels (SCL-90 scores minus depression items).

\section{Child Demographics and Mother-Informant Agreement}

In addition to the evidence that mother-reported child problems vary by level of maternal psychiatric indicators, studies have also found that the concordance between mother-informant reports of child problems varies by child demographics, such as age, sex, or race. For instance, there is some indication that depression-related elevations in maternal reports may be more likely with older children (ages 8-12 years vs. 5-7 years) (Boyle \& Pickles, 1997a). And while Renouf and Kovacs (1994) found greater concordance overall between mother-child reports of child depression symptoms as children aged, this relationship was attenuated by maternal depression: mothers with depression symptoms were more likely to provide elevated reports of child depression symptoms relative to child reports for children of any age. Children included in their study had a mean age of 11.4 years (8-13 year range).

Contradictory evidence exists for variations in report agreements by child sex. For example, using structural equation modeling with a general population sample of school-aged children, Boyle and Pickles (1997b) found a relationship between maternal depression and maternal reporting errors for symptoms of conduct disorder or hyperactivity in boys, but not girls. On the other hand, using hierarchical regression with a community sample of school-aged children (enriched to include children with behavior problems), Briggs-Gowan et al. (1996) found that, compared to child or teacher reports, anxious and depressed mothers reported elevated oppositional defiant, conduct, and attention deficit disorder symptoms in girls but not boys. Although both studies 
used similar age groups, there were differences in samples, outcome measures, and analyses which could explain differences in results.

Studies using non-clinical samples of mothers have explored the impact of child race/ethnicity on child-behavior agreement levels between mothers and others. Youngstrom et al. (2000) reported that teachers rated African-American teenagers slightly higher on externalizing behaviors than caregivers (comprised mostly of mothers), and Kolko and Kazdin (1993) found no variation in mother-child or mother-teacher agreement for reports of child problems by child race. Overall, the research evidence appears insufficient to make conclusive statements as to the differential effects of child age, gender or race on mother-rater agreement about child behavior problems.

\section{Summary and Purpose of the Current Study}

Overall, although depression appears related to elevated maternal reports of child problems relative to reports of children, teachers, or independent observers, the research to date presents several limitations: First, typically this research has used checklist measures of depression with overall population samples. Research on whether these findings replicate among mothers with depression diagnoses is limited. Second, existing research studies provide only limited contrasts of the effects of depression vs. the effects of other mental illness diagnoses, or psychiatric symptom types or levels. Finally, research on depressionrelated elevation of child problems has not systematically controlled for child age, sex, or race, although there is at least limited evidence that these child characteristics may differentially affect maternal assessments.

From the literature reviewed, it remains unclear whether or not maternal depressive symptoms, other psychiatric symptoms, or a diagnosis of depression will be more likely to be associated with elevated maternal reports of child problems when using standardized measures. Also, it is unclear whether or not elevated maternal reports of child problems associated with depression can be replicated in highrisk samples, such as mothers diagnosed with serious mental illnesses. Consequently, the purpose of our study is to directly examine the relative contribution of maternal depression symptoms, other psychiatric symptoms, and maternal psychiatric diagnosis to elevations in motherreported child problems (in contrast to teacher reports) in a largely poor, urban, minority sample of mothers diagnosed with serious mental illness. The investigation controls for child characteristics and uses 
standardized measures of maternal pathology. The results of the study have implications for research and for clinical practice. Research on families with mental illness will benefit from information about whether/what diagnoses may elevate parents' reporting of child problems or whether parent symptom levels affecting reporting are more of an issue. Clinicians can benefit from knowing whether or not mothers' diagnoses may consistently affect their view of children's problems, or whether differential symptom levels need to be accounted for in interpreting mother reports.

\section{Hypotheses}

Based on the literature reviewed, we hypothesized as follows:

1. Controlling for child characteristics and teacher-reported child behaviors, maternal depressive diagnosis (vs. other psychiatric diagnoses) will make a significant contribution to predictions of mothers' ratings of children's problem behaviors.

2. Controlling for child characteristics, teacher-reported child behaviors, and maternal depressive diagnosis, level of maternal depressive symptoms will make a significant contribution to predictions of mothers' ratings of children's problem behaviors.

\section{METHOD}

\section{Participants}

Data presented are part of two studies with overlapping samples: a longitudinal study of mothers and a second study of their adolescent children (under age 18). The first data set (NIMH \#R01 MH54321), from the MOMS study, included women (60\% AfricanAmerican, 29\% non-Hispanic White, 8\% Hispanic and 3\% other) diagnosed with a serious mental illness who were mothers of at least one child between the ages of 4 and 16. Women were recruited from the public mental health system, using clinical records and management information systems of 12 community mental health (CMH) agencies and three inpatient psychiatric units in southeast Michigan. Out of 485 eligible women identified, 78\% ( $n=379)$ completed the initial interview, $12 \%(n=59)$ declined participation and $10 \%(n=46)$ could not be contacted or scheduled. There were 42 women with no research diagnosis-14 did not complete the diagnostic interview and 28 provided insufficient information for determination of a diagnosis. Relative, to the focus of the present report, for the completion of child measures, including the Child behavior checklist (CBCL), each mother was asked to identify a target child in her family - this was the youngest child over 4 years of age for whom the mother had care responsibility at Wave 1; there were 379 target children identified at Wave 1. 
The second dataset (NIMH \# R01 MH57495), the Pathways adolescent study, included a subset from the first study: mothers who had at least one adolescent child between ages 11 and 18 years at the time of recruitment to the second study (following Wave 3), along with this adolescent child (if both mother and child agreed to participate). Of the 237 families with a child in the eligible age range at the time, in $11 \%$ $(n=27)$ of families the teen could not be located although the parent agreed; in $13 \%$ $(n=31)$ of families, parents refused; and in 5\% $(n=11)$ of families, the youth refused. Thus 168/237 youth and mothers participated or $71 \%$ of the eligible sample from the MOMS study. The Pathways sample of adolescents was balanced by sex $(n=88$ males, 80 females). Mean youth age was 15.01 years $(\mathrm{SD}=2.05)$.

The current report is based on data from both samples: adolescents from the Pathways study who were also identified as the target child for the MOMS study. In 84 families, the target child and the Pathways study participant was the same adolescent; in other cases, the target child was not yet an adolescent. However, out of these 84 families, six of the mothers did not have Diagnostic interview schedule (DIS) diagnoses. Therefore, the sample for the present study was 78 mothers and their adolescent children.

The subset of 78 mothers used in the current study had a mean age of 41.99 years $(\mathrm{SD}=6.11)$, mean education level of 12.97 years $(\mathrm{SD}=1.75)$, and $55.1 \%$ were AfricanAmerican (vs. non-African-American). Of this subset of mothers, $14.1 \%(n=11)$ had the psychiatric diagnosis of schizophrenia, $43.6 \%(n=34)$ major depression disorder, $16.7 \%$ $(n=13)$ bipolar disorder, and 25.7\% $(n=20)$ mixed psychotic and affective disorders. Compared to the other women in the MOMS study, these mothers were significantly older (mean age 38.51 vs. mean age $35.96 ; t=3.08, p=.002$ ) and had attained significantly higher education levels (mean 12.35 years vs. mean 11.79 years; $t=1.94$, $p=.05$ ) at the time of initial recruitment for the MOMS study. However, mothers in this study did not significantly differ from those participating in the larger study by race, income, level of functioning, or psychiatric diagnosis. The 78 adolescent children in this study were 59\% male and 59\% African-American; average age was 14.53 years, $\mathrm{SD}=2.02$.

\section{Procedures}

Maternal data for the MOMS study were collected in three interview waves, over a 5 year period. Permission to contact youth was obtained at the Wave 3 maternal interview. Youth interviews occurred, on average, 6 weeks later. Youth identified a core teacher (english, math, history or science) and an alternate who knew them best. The first-nominated teacher was contacted and provided a report of adolescent behavior, on average, 5-6 months after the adolescent's interview. When the first nominated teacher was not available or did not respond, the alternate teacher was contacted. The current study uses data collected from teachers and from maternal interviews. All measures used had evidence of adequate validity and reliability.

\section{Predictor Variables and Measures}

Child demographics. Youth reported their age, sex and race.

Classroom behaviors. The 14-item Finn Student Participation Questionnaire (Finn, Pannozzo, \& Voelkl, 1995; adolescent version, provided to us by Finn, personal communication October 14, 1998) was given to core teachers to rate the frequency of seven negative behaviors (e.g., is verbally or physically abusive to the teacher) on a five-point scale $(1=$ never to $5=$ always $), M=1.80, \mathrm{SD}=.66, \alpha^{\prime}=.77$. The Finn was selected 
because it covers concrete, observable behaviors, in order to maximize the validity of teachers' responses about adolescent behavior problems.

Maternal psychiatric diagnosis and symptoms. Lifetime psychiatric diagnoses were determined at Wave 1, using modules from the DIS (Robins, Helzer, Croughan, \& Ratcliff, 1981), administered by trained interviewers and supervised by an MSW-level interview coordinator and a doctoral level, senior clinical researcher who was extensively trained on the DIS. Diagnostic determinations were based on the DIS interviewer-completed form and taped interviews, and represented a consensus of the interview coordinator and clinical researcher.

The extent of current maternal psychiatric symptoms was assessed using the Colorado Symptoms Index (Shern, Wilson, \& Coen, 1994), comprised of 14 items $(1=$ never $/$ not at all in the last year to $5=$ at least every day /most of the time $)$ that form a 9-item psychosis scale $\alpha$ and a 5-item depression scale $\alpha$. Wave 3 symptom data were utilized in the present analyses: psychosis $M=2.34$, SD $=.67, \alpha=.85$; depression $M=2.82, \mathrm{SD}=.83, \alpha=.77$.

\section{Dependent Variable: Child Behavior Checklist}

Mother-reported child behavior problems were measured using the CBCL (Achenbach, Howell, Quay, \& Conners, 1991). The CBCL items represent specific child behaviors; mothers rate the degree to which their child exhibits each behavior using a three-point scale $(0=$ not true to $2=$ very true /often true $)$. Item groupings form subscale scores for externalizing behaviors (e.g., destroys his/her own things; $M=60.14, \mathrm{SD}=9.8, \alpha=.89$ ) and internalizing behaviors (e.g., too fearful or anxious; $M=60.94$, SD $=10.99$, $\alpha=.87$ ). Wave 3 CBCL scores were used in the present analyses.

\section{Analysis Plan}

To examine the relationship between maternal psychiatric diagnosis and mother-reported child behavior problem ratings, we used hierarchical regression analysis. This statistical approach allowed us to control for variables likely to affect mothers' reports and to identify the independent and unique effect of maternal diagnosis, thereby testing the first hypothesis. Given some evidence that mother-reported child behavior problems vary by child sex, age or race, we controlled for these variables. Additionally, since previous studies have used some standard of comparison to assess whether or not maternal-reported child behavior problems were, in fact, elevated, we also included teacher-reported child behaviors in the analysis as an independent observer report of child behavior problems.

All predictors (including those serving as controls) were entered as blocks into the hierarchical regression analysis in a planned order of entry. Child demographics and teacher-reported child behaviors were entered as the first two blocks. This was followed by maternal psychiatric diagnosis in block 3 . This order of entry allowed us to assess the unique contribution of maternal diagnosis (block 3) to mothers' ratings of child behavior problems, once the influence of child characteristics and teacher reports were controlled. This analysis was performed two times-once using mother-reported child internalizing problems as the dependent variable (DV) and again using mother reported child externalizing behavior problems as the DV.

To investigate the possibility that symptom level, rather than diagnosis, accounts for the elevations in mother-reported child behavior problems (our second hypothesis), we examined whether symptoms (depression or psychotic) are associated with elevations in mother-reported child behavior problems, above and beyond any effects of diagnosis. Thus, we carried out a second set of analyses, using hierarchical regression. As in the 
first set of analyses performed, controls for child characteristics and teacher-reported child behaviors were entered as the first two blocks. Next, since we were interested in the association between maternal symptoms and mother-reported child problems independent of the effects of maternal psychiatric diagnosis, we entered diagnosis in block 3 . Then in block 4, maternal depressive symptoms were added. This allowed us to identify the independent effects of depression symptoms on the variance in motherreported child problems after the effects of child characteristics, teacher reports and maternal diagnosis had been accounted for. This regression analysis was carried out for mother-reported child internalizing problems as the DV, and then again with motherreported child externalizing problems as the DV.

Following this investigation of the relationship between depression symptoms and mother-reported child behavior problems, we performed two additional regressions to assess the relationship between maternal psychotic symptoms and mother-reported child internalizing or externalizing behavior problems. This set of regressions was identical to the set of hierarchical regressions exploring the effect of maternal depression symptoms except that maternal psychotic symptoms was entered as block 4 . Consequently, we were able to assess the additional contribution of maternal psychotic symptoms to the variance in mother-reported child internalizing or externalizing behavior problems once child characteristics, teacher report and maternal diagnosis were controlled. The final results consist of 6 hierarchical regressions.

Our sample size of 78 provided $80 \%$ power to detect, as significant at $p<.05$, the contribution of a single independent variable that explains at least $8 \%$ of the variance in the DV, adjusting for the contribution of an additional 4-5 control variables that accounted for at least $15 \%$ of the DV variance- the level observed in the current data (Hintze, 2001).

\section{RESULTS}

Correlation analyses indicated that only child race was significantly related to maternal diagnosis (depression vs. other). Children with mothers diagnosed with depression were more likely to be non-AfricanAmerican than children with mothers diagnosed with another disorder. The final models for the six hierarchical regression analyses are presented in Tables 1-3. Final block coefficients are adjusted for the effects of all other variables entered into the equation at any block. With the exception of maternal diagnosis, the statistical significance of variables when first entered did not change with the entry of additional blocks. The total variance (adjusted) explained by the final models ranges from 10 to $18 \%$.

The first three blocks are identical for all regressions. Block 1 contained child demographic control variables; none of which were significantly predictive of either child internalizing or externalizing problems. Block 2 of each regression entered teacher ratings of negative child behavior, which made a unique and significant contribution to explanation of the variance in both mother-reported child externalizing (adjusted 
$\Delta R^{2}=.14, p \leq .001$ ) and internalizing (adjusted $\Delta R^{2}=.05, p \leq .05$ ) behavior problems. Children whose teachers rated them as having more negative behaviors were also more likely to be rated by their mothers as having more externalizing ( $\beta=.39$ ) or internalizing behavior problems $(\beta=.23)$ when compared to children whose teachers rated them as having fewer negative behaviors. Block 3 contained maternal diagnosis. As can be seen in Table 1, while controlling for child characteristics and teacher report of negative behavior, maternal diagnosis did not account

TABLE 1

Hierarchical Regression: Predicting Mother-Reported Child Problem Behaviors (Internalizing or Externalizing) Using Maternal Diagnosis as a Predictor While Controlling for Child Characteristics, Teacher Report $(N=78)$

\begin{tabular}{|c|c|c|c|c|}
\hline \multirow[b]{2}{*}{ Block } & \multicolumn{4}{|c|}{ Final model } \\
\hline & $\beta$ & $t$ & $\begin{array}{c}\text { Adjusted } \\
R^{2}\end{array}$ & $\begin{array}{c}\text { Adjusted } \\
\Delta R^{2}\end{array}$ \\
\hline \multicolumn{5}{|c|}{ Dependent Variable: Child Externalizing } \\
\hline \multicolumn{3}{|c|}{ 1. Child characteristics } & 0.01 & 0.01 \\
\hline Age & -0.13 & -1.24 & & \\
\hline Race $($ African-American $=1)$ & 0.00 & 0.01 & & \\
\hline $\operatorname{Sex}($ male $=1)$ & -0.00 & -0.01 & & \\
\hline 2. Teacher report (FINN negative) & 0.39 & $3.58 * * *$ & 0.15 & $0.14 * * *$ \\
\hline $\begin{array}{l}\text { 3. Maternal diagnosis } \\
(\text { depression }=1)\end{array}$ & 0.10 & 0.90 & 0.15 & 0.00 \\
\hline \multicolumn{5}{|c|}{ Dependent Variable: Child Internalizing } \\
\hline \multicolumn{3}{|c|}{ 1. Child characteristics } & 0.04 & 0.04 \\
\hline Age & -0.12 & -1.04 & & \\
\hline Race $($ African-American $=1)$ & -0.15 & -1.33 & & \\
\hline Sex $($ male $=1)$ & -0.01 & -0.06 & & \\
\hline 2. Teacher report (FINN negative) & 0.23 & $2.12^{*}$ & 0.09 & $0.05 *$ \\
\hline $\begin{array}{l}\text { 3. Maternal diagnosis } \\
(\text { depression }=1)\end{array}$ & 0.17 & 1.50 & 0.10 & 0.01 \\
\hline
\end{tabular}

Note. Beta coefficients presented are standardized.

$* p \leq .05$. *** $p \leq .001$.

$* p \leq .05$. *** $p \leq .001$. 
for additional variance in mother-reported child externalizing (adjusted $\Delta R^{2}=.00, \mathrm{NS}$ ) or internalizing (adjusted $\Delta R^{2}=.01, \mathrm{NS}$ ).

It is important to note that preliminary regression analyses that controlled for teacher-reported negative child behaviors but not for child demographics (race, sex, and age), resulted in a significant, independent contribution for maternal diagnosis predicting motherreported child internalizing scores (Adjusted $\Delta R^{2}=.04, p \leq .05$ ) and externalizing scores (Adjusted $\Delta R^{2}=.02, p \leq .05$ ). Without controlling for the potentially confounding effects of child demographic variables, children whose mothers were diagnosed with depression appeared more likely to be rated by their mothers as having higher internalizing $(\beta=.22)$ or externalizing $(\beta=.17)$ behavior scores. This effect was not found when the effects of child demographic variables were controlled.

The regressions presented in Table 2 add maternal depression symptoms in block 4 to the 3-block equation displayed in Table 1. Maternal depression symptoms made a unique and significant contribution to explaining the variance in mother-reported child internalizing problems (adjusted $\Delta R^{2}=.04, p \leq .05$ ), but not to mother-reported child externalizing problems (adjusted $\Delta R^{2}=.01$, NS). Regarding the former, children whose mothers reported more depression symptoms were rated by their mothers as having higher internalizing behavior problems $(\beta=.23)$ compared to children whose mothers reported fewer depression symptoms.The findings for the third set of hierarchical regressions can be found in Table 3. In these equations, maternal psychosis symptoms were entered in block 4 in place of depression symptoms. Maternal psychosis symptoms made a unique and significant contribution to explaining the variance in both mother-reported child externalizing (adjusted $\Delta R^{2}=.03, p \leq .05$ ) and mother-reported child internalizing problems (adjusted $\Delta R^{2}=.07, p \leq .05$ ). Children whose mothers reported more psychosis symptoms were rated by their mothers as having higher levels of externalizing $(\beta=.21)$ and internalizing ( $\beta=.28$ ) behavior problems when compared to children whose mothers reported fewer psychosis symptoms.

\section{DISCUSSION}

The present study examined the relative contribution of maternal psychiatric diagnosis and of maternal depression or psychotic symptoms to elevations in mother-reported child problems in a largely poor, urban, minority sample of mothers diagnosed with serious mental ill- 
nesses. We used standardized measures of maternal clinical variables and, through hierarchical regression techniques, controlled for child characteristics and teacher reports of children's negative behaviors. A finding that was consistent across all analyses was that high negative teacher ratings did relate to high mother-reported child behavior problems, and that a significant amount of the variance in mother-

TABLE 2

\section{Hierarchical Regression: Predicting Mother-Reported Child Problem Behaviors (Internalizing or Externalizing) Using Maternal Depression Symptoms as a Predictor While Control- ling for Child Characteristics, Teacher Report, and Maternal Diagnosis $(\boldsymbol{N}=78)$}

\begin{tabular}{lcccc}
\hline & \multicolumn{3}{c}{ Final model } \\
\cline { 2 - 4 } Block & & & Adjusted & Adjusted \\
\hline & & $t$ & $R^{2}$ & $\Delta R^{2}$ \\
\hline
\end{tabular}

Dependent Variable: Child Externalizing

1. Child characteristics

Age $-0.14-1.28$

Race (African-American = 1)

$\begin{array}{ll}0.06 & 0.48\end{array}$

Sex $($ male $=1)$

$0.03 \quad 0.24$

2. Teacher report (FINN negative)

$0.38 \quad 3.50^{* * * *}$

0.01

0.01

3. Maternal diagnosis

$0.13 \quad 1.17$

0.15

$0.14^{* * *}$ (depression $=1)$

4. Maternal depression symptoms

$0.16 \quad 1.37$

0.15

0.00

Dependent Variable: Child Internalizing

1. Child characteristics

0.04

0.04

Age

Race (African-American $=1$ )

Sex $($ male $=1)$

2. Teacher report (FINN negative)

$-0.12-1.10$

$-0.07-0.60$

$0.04 \quad 0.32$

0.222 .03

3. Maternal diagnosis

0.221 .91 $($ depression $=1)$

4. Maternal depression symptoms

$0.232 .00^{*}$

0.14

0.09

$0.05^{*}$

0.10

0.01

0.01

$0.16 \quad 0.01$

Note. Beta coefficients presented are standardized.

$* p \leq .05$. *** $p \leq .001$.

$* p \leq .05$. *** $p \leq .001$. 
TABLE 3

Hierarchical Regression: Predicting Mother-Reported Child Problem Behaviors (Internalizing or Externalizing) Using Maternal Psychosis Symptoms as a Predictor While Controlling for Child Characteristics, Teacher Report, and Maternal Diag$\operatorname{nosis}(\boldsymbol{N}=78)$

\begin{tabular}{|c|c|c|c|c|}
\hline \multirow[b]{2}{*}{ Block } & \multicolumn{4}{|c|}{ Final model } \\
\hline & $\beta$ & 1 & $\begin{array}{l}\text { Adjusted } \\
R^{2}\end{array}$ & $\begin{array}{l}\text { Adjusted } \\
\Delta R^{2}\end{array}$ \\
\hline \multicolumn{5}{|c|}{ Dependent Variable: Child Externalizing } \\
\hline \multicolumn{3}{|c|}{ 1. Child characteristics } & 0.01 & 0.01 \\
\hline Age & -0.13 & -1.27 & & \\
\hline Race (African-American = 1) & 0.04 & 0.35 & & \\
\hline Sex $($ male $=1)$ & 0.16 & 0.15 & & \\
\hline 2. Teacher report (FINN negative) & 0.39 & $3.69 * * *$ & 0.15 & $0.14 * * *$ \\
\hline $\begin{array}{l}\text { 3. Maternal diagnosis } \\
(\text { depression = } 1)\end{array}$ & 0.15 & 1.31 & 0.15 & 0.00 \\
\hline 4. Maternal psychotic symptoms & 0.21 & $2.00^{*}$ & 0.18 & $0.03 *$ \\
\hline \multicolumn{5}{|c|}{ Dependent Variable: Child Internalizing } \\
\hline \multicolumn{3}{|c|}{ 1. Child characteristics } & 0.04 & 0.04 \\
\hline Age & -0.12 & -1.09 & & \\
\hline Race $($ African-American $=1)$ & -0.10 & -0.92 & & \\
\hline Sex $($ male $=1)$ & 0.02 & 0.16 & & \\
\hline 2. Teacher report (FINN negative) & 0.24 & $2.25^{*}$ & 0.09 & $0.05^{*}$ \\
\hline $\begin{array}{l}\text { 3. Maternal diagnosis } \\
(\text { depression }=1)\end{array}$ & 0.23 & $2.06^{*}$ & 0.10 & 0.01 \\
\hline 4. Maternal psychotic symptoms & 0.28 & $2.58 *$ & 0.17 & $0.07 *$ \\
\hline
\end{tabular}

Note. Beta coefficients presented are standardized.

$* p \leq .05 . * * * p \leq .001$.

$* p \leq .05$. ***p $\leq .001$.

reported child behavior problems (i.e., 5-14\%) can be understood in terms of behaviors that teachers observe in the child.

Our first hypothesis was that maternal depression diagnosis would predict elevations in mother-reported child problems; it was not supported. Controlling for child characteristics and teacher-reported negative child behavior, children with mothers diagnosed with 
depression were not more likely than those whose mothers had another psychiatric diagnosis to be given higher mother-reported problem ratings. Differences in our findings compared to those of others appear to be primarily related to our use of controls for child demographics; that is, a preliminary analysis of our data did find a relationship between maternal diagnosis and child problems without controls.

The absence of support for the first hypothesis is consistent with other studies with smaller clinical samples (Hammen et al., 1987; Conrad \& Hammen, 1989) and from community samples (Richters \& Pellegrini, 1989), using a variety of diagnostic techniques. Our results suggest that these findings may be generalizeable to high-risk samples because our study used a standardized diagnostic instrument with a large, clinical sample of mothers who were predominantly from racial minority and lower socioeconomic groups. Also, our study controlled for variables which were not systematically included in other studies (child age, sex and race). Some studies with findings consistent with ours did not use child demographics as controls either; however, they were comprised of less diverse samples (e.g., predominantly white). Our results highlight the importance of using child demographics as controls when including participants from diverse racial and socioeconomic backgrounds.

Diagnosis of major depression may be unrelated to elevated motherreported child behavior problems in this sample because diagnosis is poorly related to functioning in cases of serious mental illness, as demonstrated by research in psychiatric rehabilitation (Tsang, Lam, $\mathrm{Ng}$, \& Leung, 2000). That is, diagnosis is likely to remain constant despite fluctuations in symptoms which may be more directly related to maternal behavior and/or perceptions. Lifetime diagnosis, as a historical variable, does not reflect the known variability in functioning displayed by people with serious mental illnesses, the heterogeneity of symptoms within diagnostic groups, the overlap of symptoms across diagnostic categories, or confounds between diagnosis and other variables, such as race.

Although depression diagnosis was not significant when added to the model after controls, it became significant when psychotic symptoms were added to the regression model. It appears that this result reflects a suppressor effect. That is, the addition of psychotic symptoms to the equation accounts for the variance in depression diagnosis that is unrelated to maternal rating of child internalizing problems. Removing the psychosis/no psychosis component from the diagnosis variable leaves within the depression diagnosis variable the variance related to the affective component. By reducing the irrelevant (i.e., error) 
variance in depression diagnosis, the addition of psychotic symptoms increases the size of the explanatory effect of diagnosis, relative to its error variance, and causes the contribution of depression diagnosis to become significant (Cohen, Cohen, West, \& Aiken, 2003). Thus, a depression diagnosis may act as a proxy for symptoms, when they are not measured directly.

Some support was provided for the second hypothesis: that maternal symptoms of major depression would relate to elevated motherreported child problems independent of teacher report, child demographics, and maternal psychiatric diagnosis. Greater maternal depression symptoms predicted higher internalizing scores above and beyond child characteristics, teacher report and maternal diagnosis. This finding is consistent with some research (Youngstrom et al., 2000) which found that caregiver depression was associated with elevations in caregiver-reported CBCL internalizing behaviors, relative to teacher report.

Our study did not find a relationship between maternal depression symptoms and elevated mother ratings of child externalizing, in contrast to Boyle and Pickles (1997b). It is possible that differences in results were due to sample and measurement differences. For example, the study by Boyle and Pickles (1997b) included a population sample and specifically measured conduct and hyperactivity problems using both CBCL and DSM-III items. We, on the other hand, recruited a clinical sample of mothers and measured child externalizing problems using only the CBCL. Youngstrom et al. (2000) also found a relationship between caregiver depression and caregiver-reported CBCL externalizing ratings, relative to teacher report. However, they used a community sample comprised solely of male youth. Finally, in our study, externalizing behaviors may not have related to maternal symptoms because they are often more obvious and/or bothersome and less likely to be influenced by mothers' symptoms or perceptions.

Maternal psychotic symptoms were related to higher maternal reports of both externalizing and internalizing behavior problems, even after controlling for child demographics and teacher-reported negative child behaviors. This finding is consistent with research in which psychiatric symptoms other than depression have significantly related to elevated mother-reported child behavior problems, such as anxiety (Chilcoat \& Breslau, 1997; Frick et al., 1994). On the other hand, Kolko and Kazdin (1993) found that higher general psychiatric symptom levels of parents, using the SCL-90, did not predict parent-teacher disagreements regarding child problems. Although these findings differed from ours, this may be due to differences in methodology such as the sample used 
(i.e., they recruited children from a larger study comprised of a mixed community and clinical sample and children in their sample were younger with a mean age of 9.1 years). It is also possible that elevated maternal reports of child behavior problems are only associated with specific psychotic symptom items, such as those directly impacting cognitive appraisals (e.g., psychotic thinking) rather than other functions (e.g., vegetative symptoms such as loss of appetite). Different symptom measures might therefore produce differing results.

Limitations of this study include the fact that teacher and mother reports were from different contexts and that measurements occurred at different time points (up to 6 months apart). Also, the validity of the teachers' reports could be questioned, since children might be likely to nominate teachers with whom they had better relationships, which could differentially affect teacher ratings. It should be noted, however, that child nominations are frequently used to select teachers most informed to do student ratings. Moreover, this study is unable to provide the information needed to determine if the mother-reported elevations in child problems are the result of a maternal bias (Najman et al., 2001), as a comprehensive, objective expert assessment of the child across multiple contexts was not available. Further, we need to acknowledge the possibility that children may indeed behave differently in the context of their mothers' highly symptomatic presentation. Finally, interpretation of results might have been clearer were we able to compare the effects of psychotic vs. depressive symptoms in the same regressions. Unfortunately, this was not possible, given the fact that the two symptom measures were highly correlated.

In sum, our study failed to provide support for the hypothesis that maternal diagnosis of depression is significantly related to elevated mother-reported child behavior problems. Findings inconsistent with the current results appear to be largely due to differences in methodology. That is, our study findings indicate that it is important to systematically control for child demographics in such analyses. Further, although maternal and teacher report of child problems are generally related, when mothers have more psychiatric symptoms, including depressive symptoms, they report more child behavior problems. This finding supports our second hypothesis and indicates that future research studies should assess mothers' symptom levels, as well as diagnosis; depression diagnosis is not the equivalent of symptomatic impairments, although diagnosis may act as a proxy for this variable when specific symptom measures are not available. In a service delivery context, clinicians need not assume that mothers with a depression diagnosis are likely to 
over-report children's problems. Rather, when mothers with a serious mental illness report distress over their children's behaviors, the woman's current symptomatology is more likely to relate to reports of more child behavior problems than is her diagnosis of depression.

\section{REFERENCES}

Achenbach, T. M., Howell, C. T., Quay, H. C., \& Conners, C. K. (1991). National survey of problems and competencies among 4 to 16 year-olds: Parents reports for normative and clinical samples. Monographs of the Society for Research in Child Development, 56(3), R5-R119.

Ackermann, R., \& DeRubeis, R. J. (1991). Is depressive realism real? Clinical Psychology Review, 11(5), 565-584.

Boyle, M. H., \& Pickles, A. (1997a). Influence of maternal depressive symptoms on ratings of childhood behavior. Journal of Abnormal Child Psychology, 25(5), 399-412.

Boyle, M. H., \& Pickles, A. (1997b). Maternal depressive symptoms and ratings of emotional disorder symptoms in children and adolescents. The Journal of Child Psychology and Psychiatry and Allied Disciplines, 38(8), 981-992.

Briggs-Gowan, M. J., Carter, A. S., \& Schwab-Stone, M. (1996). Discrepancies among mother, child, and teacher reports: Examining the contributions of maternal depression and anxiety. Journal of Abnormal Child Psychology, 24(6), 749-765.

Chilcoat, H. D., \& Breslau, N. (1997). Does psychiatric history bias mothers' reports? An application of a new analytic approach. Journal of the American Academy of Child and Adolescent Psychiatry, 36(7), 971-979.

Cohen, J., Cohen, P., West, S. G., \& Aiken, L. S. (2003). Applied multiple regression/correlation analysis for the behavioral sciences, (3rd ed) Mahweh, NJ: Erlbaum.

Conrad, M., \& Hammen, C. (1989). Role of maternal depression in perceptions of child maladjustment. Journal of Consulting and Clinical Psychology, 57(5), 663-667.

Fergusson, D. M., Lynskey, M. T., \& Horwood, L. J. (1993). The effect of maternal depression on maternal ratings of child behavior. Journal of Abnormal Child Psychology, 21(3), 245-269.

Finn, J. D., Pannozzo, G. M., \& Voelkl, K. E. (1995). Disruptive and inattentive-withdrawn behavior and achievement among $4^{\text {th }}$ graders. Elementary School Journal, 95(5), 421-434.

Frick, P. J., Silverthorn, P., \& Evans, C. (1994). Assessment of childhood anxiety using structured interviews: Patterns of agreement among informants and association with maternal anxiety. Psychological Assessment, 6(4), 372-379.

Haaga, D. A. F., \& Beck, A. T. (1995). Perspectives on depressive realism: Implications for cognitive theory of depression. Behavior Research and Therapy, 33(1), 41-48.

Hammen, C., Adrian, C., Gordon, D., Burge, D., Jaenicke, C., \& Hiroto, D. (1987). Children of depressed mothers: Maternal strain and symptom predictors of dysfunction. Journal of Abnormal Psychology, 96((3), 190-198.

Hintze, J. (2001). NCSS and PASS 2002, Kaysville, UT: Number Cruncher Statistical Systems.

Kolko, D. J., \& Kazdin, A. E. (1993). Emotional/behavioral problems in clinic and nonclinic children: Correspondence among child, parent, and teacher reports. Journal of Child Psychology and Psychiatry, 34(6), 991-1006.

Najman, J. M., Williams, G. M., Nikles, J., Spence, S., Bor, W., O’Callaghan, M., LeBrocque, R., \& Andersen, M. J. (2000). Mothers' mental illness and child behavior problems: Cause-effect association or observation bias? Journal of the American Academy of Child and Adolescent Psychiatry, 39(5), 592-602.

Najman, J. M., Williams, G. M., Nikles, J., Spence, S., Bor, W., O’Callaghan, M., LeBrocque, R., Andersen, M. J., \& Shuttlewood, G. J. (2001). Bias influencing maternal reports of child behaviour and emotional state. Social Psychiatry and Psychiatric Epidemiology, 36, 186-194.

Querido, J. G., Eyberg, S. M., \& Boggs, S. R. (2001). Revisiting the accuracy hypothesis in families of young children with conduct problems. Journal of Clinical Child Psychology, 30(1), 253-261.

Renouf, A. G., \& Kovacs, M. (1994). Concordance between mothers' reports and children's selfreports of depressive symptoms: A longitudinal study. Journal of the American Academy of Child and Adolescent Psychiatry, 33(2), 208-216. 
Richters, J., \& Pellegrini, D. (1989). Depressed mothers' judgments about their children: An examination of the depression-distortion hypothesis. Child Development, 60, 1068-1075.

Robins, L. N., Helzer, J. E., Croughan, J., \& Ratcliff, K. S. (1981). National Institute of Mental Health Diagnostic Interview Schedule: Its history, characteristics, and validity. Archives of General Psychiatry, 38(4), 381-389.

Sawyer-Radloff, L. (1977). The CES-D Scale: A self-report depression scale for research in thegeneral population. Applied Psychology Measurement, 1(3), 385-401.

Shern, D. L., Wilson, N. Z., \& Coen, A. S. (1994). Client outcomes II: Longitudinal client data from the Colorado Treatment Outcome Study. Milbank Quarterly, 72, 123-148.

Treutler, C. M., \& Epkins, C. C. (2003). Are discrepancies among child, mother, and father reports on children's behavior related to parents' psychological symptoms and aspects of parent-child relationships? Journal of Abnormal Child Psychology, 31(1), 13-27.

Tsang, H., Lam, P., Ng, B., \& Leung, O. (2000). Predictors of employment outcome for people with psychiatric disabilities: A review of the literature since the mid ' $80 \mathrm{~s}$. Journal of Rehabilitation, 66(2), 19-31.

Youngstrom, E., Izard, C., \& Ackerman, B. (1999). Dysphoria-related bias in maternal ratings of children. Journal of Consulting and Clinical Psychology, 67(6), 905-916.

Youngstrom, E., Loeber, R., \& Stouthamer-Loeber, M. (2000). Patterns and correlates of agreement between parent, teacher, and male adolescent ratings of externalizing and internalizing problems. Journal of Consulting and Clinical Psychology, 68(6), 1038-1050.

\section{IN MEMORIAM: CAROL T. MOWBRAY, Ph.D.}

As many of you of aware, an important and much revered member of the mental health services research community, Dr. Carol Mowbray, passed away on August 23, 2005 after a brief bout with cancer. Carol's main research activities were in the areas of social integration and recovery, focusing on persons with severe and persistent mental illness, in particular homeless individuals and those with co-occurring substance abuse disorders. Much of her work was carried out within a recovery / rehabilitation framework and was guided by the belief that services and delivery systems could best improve the lives of persons with psychiatric disabilities by removing obstacles to their integration and by creating supports for their efforts at recovery. The viability and vitality of psychiatric rehabilitation as a research domain owe much to Carol's substantive contributions, her collegiality, and her willingness to be a mentor.

There already have been many and certainly will be more tributes to the quality and importance of Carol's research. I have been invited by the Journal to write a memorial note for Carol in my role as the chair of the Mental Health Section of the American Public Health Association. Writing from that perspective, I would like to emphasize another aspect of Carol's career that might be overlooked-the career itself. To many, Carol is known principally as a professor of social work at an elite university. But those of us who have worked in public mental health since the 1980s knew her first when she served as Director of Research for the Michigan Department of Mental Health. In that role she was among a handful of pioneering researchers working within state departments of mental health across the nation, seeking to demonstrate the potential contributions research could make to the delivery of effective services. Many who are now among the leaders in the field of mental health services research and who work in academic settings-a group in which Carol figured prominently-began their careers in this way, and brought the notion of a "public health mission" with them when they moved from state government to academia. I would like to take this opportunity to encourage young services researchers to embrace that same public health perspective as they consider their own career paths. In doing so, they could find no better a guide than the example left by Carol Mowbray.

I am grateful to the Community Mental Health Journal for the opportunity to emphasize this important aspect of Carol's work and life on behalf of the Mental Health Section.

William H. Fisher, Ph.D. Chair, Mental Health Section American Public Health Association

Professor of Psychiatry
University of Massachusetts Medical School
Worcester, Massachusetts

